\title{
A Proposal Tool for Immersive Microbiology Teaching Tool
}

\author{
Larissa Fernandes ${ }^{\dagger}$ \\ Programa de Pós-graduação em Ensino \\ Universidade Tecnológica Federal do Paraná \\ larissa.1999@alunos.utfpr.edu.br \\ ORCID: https://orcid.org/0000-0003-3257-6381
}

\author{
Eduardo Filgueiras Damasceno \\ Programas de Pós-Graduação em Ensino e Informática \\ Universidade Tecnológica Federal do Paraná \\ damasceno@utfpr.edu.br \\ ORCID: http://orcid.org/0000-0002-6246-1246
}

\begin{abstract}
There were several ways to teaching microbiology, but when it is hands-on into laboratory practice, biosafety must take priority. To mitigate this limitation, some approaches use virtual reality technology to simulate the environment. However, all are shows as multimedia-based, didactic simulators and function as an extension of the textbook. This short paper addresses a proposal to develop a virtual reality environment for teaching microbiology that can be used as a tool to explore the microbiological environment for the study of viruses and bacteria harmful to humans. Finally, this work aims to develop a prototype tool to facilitate the teaching of microbiology, contemplating both the pedagogical requirements of the educational software and the technical requirements needed to build an immersive virtual reality environment. Added to this proposal is the requirement to present a low-cost tool for both editing and visualization.
\end{abstract}

\section{CCS CONCEPTS}

Applied computing $\rightarrow$ Life and medical sciences $\rightarrow$ Systems biology

\section{KEYWORDS}

Virtual Reality, Learning Environment, Low-Cost, Microbiology

\section{Introduction}

We are amid ever-increasing technological advances, where every decade is moving further into the digital age. Technological impulses have transformed in all fields and go beyond the imagination of previous generations. So have innovative educational applications, going beyond the scope of learning expectations and hovering over the objectivity of student authorship and knowledge representation. Technology in the field of biology has changed traditional teaching and learning, increasing the awareness of teachers at all educational levels [1].

In this case, a variety of possibilities of studies on the properties of Virtual Reality (VR) glasses, mainly concerning immersion and interaction, can be explored in the development of new educational applications [2]. Hence, Immersive Virtual Reality (IVR) applications and environments use Virtual Reality (VR) digital technology and empower the user to "be" anywhere without the need to leave their current location [3].

Thus, this emerges as an alternative tool to traditional learning, providing a social and experiential way of acquiring knowledge [4], and it promotes a high level of realism in simulations and dramatizations that were not present in a teaching environment before [5]. Another advantage of these tools is that they provide students with the ability to develop some special skills: exploration, simulation, and combining concepts. These concepts are significant as they develop learning autonomy [6]. The possibilities of interaction with virtual objects in VR promote the resignification of abstract concepts. Maybe because the conversion of subjective concepts into concrete ones occurs through more engage, active, and interactive learning [7].

\section{Related Work}

The VR laboratory is considered a teaching strategy that is increasingly being used in several areas of health care for professional skills training and assessment as well as chemistry courses, biotech education, genetics, and a wide variety of other fields [8]. These virtual environments provide an extensive learning platform that not only adds to student learning but can also increase their motivation to learn and improve their self-efficacy in final exams when compared with a traditional lecture [9].

Tus, these simulations have been shown to improve the achievement of learning outcomes among students, by making the learning experience more immersive and engaging. CellVerse is a VR [10] environment designed for the classroom to give the student an intense and evocative experience of the biological environment as seen from the inside. Designed to give the student the feature of selecting and manipulating the biological images and objects displayed on a screen, it motivates the student to discover more about the element being touched.

The $360^{\circ}$ VR tour is very useful to familiarise the student and immerse them in the context. This proposal proved to be attractive and motivating because it abuses colorful objects and demonstration of microbiological life through predefined animations. We observed the key to a successful VR experience is developing an intuitive environment that allows the user to explore the environment (data) at their own pace, while also guiding them so they do not get lost or overwhelmed by the experience. In addition, most of the work reports the need for the guide, both for the $360^{\circ}$ tour and for the spontaneous navigation and exploration, with the goal of the student not getting lost from the class in an area of the 3D environment without attention to the content presented. So, other papers present a more instrument-based approach to microbiology. These use the microscope to present cells, bacteria, and microorganisms simulated in computer graphics. Thus, Onlabs 
is a VR biology lab designed to offer a high level of realism regarding microscopy [11]. This virtual lab is designed to help students understand the flow of the experiment while learning the details of each step at the same time. In each laboratory experiment, the student learns about each instrument and how to apply it in biochemical study.

\section{Proposed Solution}

The Application was developed at UTFPR's Visual Computing Laboratory was designed to work on two different platforms: One of them on Windows desktop and another on Android Mobile. The desktop version was designed to meet the teacher's needs to guide the class during an exhibition. The mobile version was developed so that students can immerse themselves in the simulated universe created.

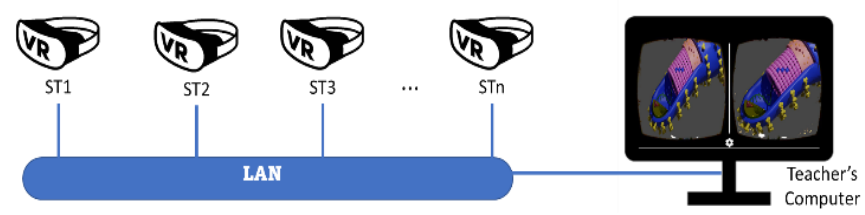

Fig. 1. VRBio Comunication Layer

In Fig. 1 we see the illustration of VRBio in a LAN network, in which the points identified with ST (from 1 to $n$ ) are the visualization devices model CardBorad, synchronized accessing the same environment but generating different visualizations about the same object of study, controlled by the Teacher's Computer. So, Fig. 2 shows the structure of the application's interface on the desktop platform

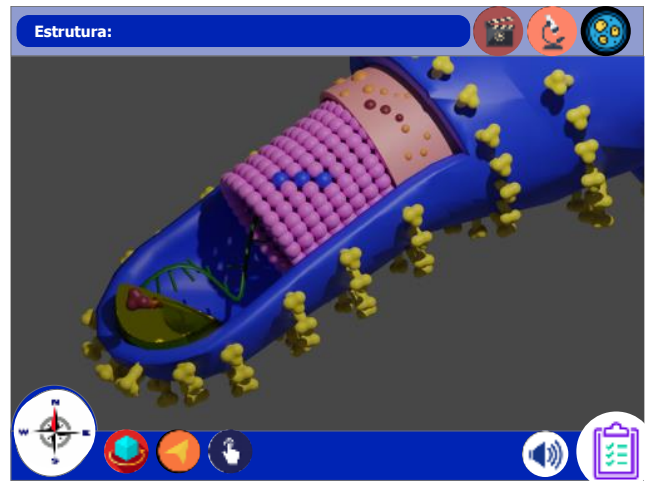

Fig. 2. VRBio Desktop Interface

The mobile version is shown in Fig. 3. This has fewer interaction objects since that is used by linking via the ray tracing algorithm. Thus, the user interacts with the 3D objects with joystick control. In the upper right region are the icons identifying the type of view (In Vivo, In Vitro, and Video-player). And at the bottom of the same side is the icon that initializes the Quiz. Likewise, in the lower-left corner, there is an icon that displays a compass so that the user does not lose the orientation of the object in the virtual environment.

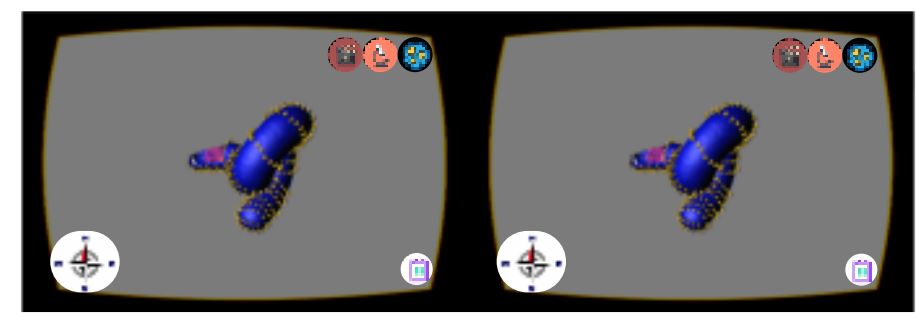

Fig. 3. VRBio Desktop Interface

\section{Final Considerations}

In this paper, we show some educational tools that are often incorporated in the learning process as self-paced learning tools that help students acquire confident knowledge of science and laboratory skills. We proposed a tool that could combine the best features of these works to be applied in the future in a real field study. We know the limitations of the teaching field, and for this reason, in addition to the release of the tool, a tutorial course on how to use it in the classroom will be considered, presenting practical cases of use in a didactic sequence.

\section{REFERENCES}

[1] J. Labovitz and C. Hubbard, "The Use of Virtual Reality in Podiatric Medical Education," Clinics in Podiatric Medicine and Surgery, vol. 37, no. 2, pp. 409 420, Apr. 2020, doi: 10.1016/j.cpm.2019.12.008.

[2] A. Makhkamova, J.-P. Exner, T. Greff, and D. Werth, "Towards a Taxonomy of Virtual Reality Usage in Education: A Systematic Review," in Augmented Reality and Virtual Reality: Changing Realities in a Dyanmica World, Springer, 2020, pp. 283-296. doi: 10.1007/978-3-030-37869-1_23.

[3] A. C. Howland, R. Rembisz, T. S. Wang-Jones, S. R. Heise, and S. Brown, "Developing a virtual assessment center," Consulting Psychology Journal, vol. 67, no. 2, pp. 110-126, 2015, doi: 10.1037/cpb0000034.

[4] H. M. Huang and S. S. Liaw, "An analysis of learners' intentions toward virtual reality learning based on constructivist and technology acceptance approaches," International Review of Research in Open and Distance Learning, vol. 19, no. 1, pp. 91-115, 2018, doi: 10.19173/irrodl.v19i1.2503.

[5] B. $\mathrm{Wu}, \mathrm{X} . \mathrm{Yu}$, and $\mathrm{X} . \mathrm{Gu}$, "Effectiveness of immersive virtual reality using head-mounted displays on learning performance: A meta-analysis," British Journal of Educational Technology, vol. 51, no. 6, pp. 1991-2005, 2020, doi: 10.1111/bjet.13023.

[6] J. Chambers, The Digital Transformation of Europe:Connecting Schools, Empowering Learners, no. September. UNESCO / UNICEF, 2020.

[7] C. Celik, G. Guven, and N. K. Cakir, "Integration of Mobile Augmented Reality (MAR) Applications into Biology Laboratory: Anatomic Structure of the Heart," Research in Learning Technology, vol. 28. 2020.

[8] G. Makransky, M. W. Thisgaard, and H. Gadegaard, "Virtual simulations as preparation for lab exercises: Assessing learning of key laboratory skills in microbiology and improvement of essential non-cognitive skills," PLOS ONE, vol. 11, no. 6, p. e0155895, Jun. 2016, doi: 10.1371/journal.pone.0155895.

[9] N. S. Nowlan, P. Hartwick, and A. Arya, "Skill assessment in virtual learning environments," CIVEMSA 2018 - 2018 IEEE International Conference on Computational Intelligence and Virtual Environments for Measurement Systems and Applications, Proceedings, 2018, doi: 10.1109/CIVEMSA.2018.8439968.

[10] A. Wang et al., "Iterative user and expert feedback in the design of an educational virtual reality biology game," Interactive Learning Environments, vol. 0, no. 0, pp. 1-18, 2019, doi: 10.1080/10494820.2019.1678489.

[11] E. Paxinou, C. T. Panagiotakopoulos, A. Karatrantou, D. Kalles, and A. Sgourou, "Implementation and Evaluation of a Three-Dimensional Virtual Reality Biology Lab versus Conventional Didactic Practices in Lab Experimenting with the Photonic Microscope," Biochemistry and Molecular Biology Education, vol. 48, no. 1, pp. 21-27, Jan. 2020, doi: 10.1002/bmb.21307. 